\title{
EMERGÊNCIA DAS PESQUISAS SOBRE SAÚDE E MEIO AMBIENTE NO BRASIL: A CONTRIBUIÇÃO DOS PROGRAMAS DE PÓS-GRADUAÇÃO
}

The necessity of researches on environment and health in Brazil: the contribution of post-graduate programs

\author{
Sônia Beatris Balvedi Zakrzevski1'; Marilize Pereira²; Arnaldo Nogaro³; \\ Albanin Aparecida Mielniczk Pereira ${ }^{4}$
}

\footnotetext{
${ }^{1}$ Graduada em Ciências. Mestre em Educação e Doutora em Ecologia. Professora do PPG em Ecologia, URI, Erechim. URI Erechim. Lab. de Educação Ambiental.

${ }^{2}$ Graduada em Enfermeira. Mestre em Ecologia. Enfermeira do Instituto Federal de Sertão, RS

${ }^{3}$ Graduado em Filosofia. Mestre em Antropologia Filosófica e Doutor em Educação. Professor do PPG em Educação, URI Frederico Westphalen

${ }^{4}$ Graduada em Biologia. Mestre em Biologia Funcional e Molecular e Doutora em Biologia Celular e Molecular. Professora do PPG Ecologia, URI, Erechim
}

RESUMO: A relação entre saúde e meio ambiente tem se tornado cada vez mais evidente, passando a fazer parte das diretrizes de políticas públicas do Brasil. Um dos desafios neste cenário é a aproximação dos grupos acadêmicos com os profissionais da área de saúde ambiental. Por isso, é importante conhecer a dinâmica das pesquisas acadêmicas, sobre esta temática, realizadas no País. Neste estudo foram mapeadas as dissertações e teses sobre saúde e meio ambiente, elaboradas no Brasil entre 1987 e 2010. Os resultados mostram que houve um crescimento exponencial dessas produções, realizadas por programas de pós-graduação (PPG), de diferentes áreas do conhecimento, com ênfase para as áreas multidisciplinar e ciências da saúde. Os estudos foram produzidos, principalmente, pelos PPG vinculados às instituições federais e estaduais, localizadas na região sudeste do Brasil. Os dados indicam a necessidade de descentralização das pesquisas, visando a atender às demandas nacionais e regionais de integração entre educação, ambiente e saúde. 
Palavras-chave: Saúde ambiental. Trabalhos acadêmicos. Pesquisa bibliográfica.

\begin{abstract}
The relationship between health and environment has become increasingly evident, becoming part of the Brazil public policy guidelines. One of the challenges in this scenario is group closing between academic groups and environmental health professionals. Therefore, it is important to know the dynamics of academic research on health and environment which is carried out in the country. In this study, dissertations and theses on environment and health, produced between 1987 and 2010, were mapped. The results show that there was an exponential growth of these academic productions carried out by degree programs of different areas of knowledge, with emphasis on multidisciplinary areas and health sciences. The studies were produced mainly by educational institutions linked to federal and state organizations, located in the Southeastern region of Brazil. The data pointed out the necessity of research decentralization, aiming to meet national and regional demands for education, environment and health integration.
\end{abstract}

Keywords: Environmental Health. Dissertations Academic. Bibliographic research.

\section{Introdução}

A consciência humana sobre a influência do ambiente na saúde é bastante antiga. Entretanto, essa concepção se ampliou nos séculos XVIII e XIX, com o aumento da industrialização e da urbanização. Nesse contexto, as intervenções sanitárias relacionavam-se com a teoria dos miasmas, que acreditava que partículas de putrefação presentes no ar eram responsáveis pela disseminação de doenças e, por essa razão, deveriam ser eliminadas. A higiene foi introduzida para controlar os miasmas, seja nos locais de trabalho, quanto nos espaços urbanos e nas residências (MINAYO, 2012).

Atualmente, entende-se que saúde e meio ambiente são áreas intrinsecamente relacionadas, não sendo possível proteger e prevenir a saúde individual e/ou coletiva sem termos o cuidado com o meio ambiente, ou seja, saúde pressupõe um meio ambiente saudável. Da mesma forma, não se pode falar em danos ao meio ambiente sem pensar, concomitantemente, em danos à saúde individual e à coletiva. Diferentes estudos têm demonstrado que tanto os contaminantes ambientais quanto as mudanças globais no ecossistema podem influenciar, direta ou indiretamente, a saúde das populações humanas (CARTER -POKRAS et al., 2007; MYERS et al., 2013; REDSHAW et al., 2013).

Bastos (2011) alerta que a progressiva deterioração das condições ambientais do planeta tem relação evidente com a saúde. Seguimos construindo casas imensas para nossa proteção, como se fôssemos uma espécie de castor gigante e insaciável. Só que, ao contrário dos animais, não nos contentamos em construir as casas, também drenamos rios e matamos não alguns, mas todos os peixes, não os predando um a um, como fazem as lontras ou as gaivotas, mas capturando-os, 
às centenas ou aos milhares, com redes, venenos e bombas. Com isso, comprometemos a integridade dos ecossistemas e a segurança alimentar e a imensa população humana, que, estima-se, ultrapassará 9 bilhões em 2050, se de fato chegarmos até lá.

Segundo a Organização Pan-Americana de Saúde (OPS, 2010), a relação entre o ambiente e o padrão de saúde da população define a saúde ambiental, incorporando os fatores que, potencialmente, afetam a saúde, como substâncias químicas, elementos biológicos, situações que interferem no estado psíquico do indivíduo, bem como os aspectos negativos do desenvolvimento social e econômico dos países.

A interface saúde-meio ambiente pode ser explorada por uma grande diversidade de áreas do conhecimento, incluindo desde as ciências biológicas até as engenharias (VEIGA; FERNANDES, 1999; BUSS, 2000; CONFALONIERI, 2005; GOMES; ESTEVES, 2012; TELES et al., 2013). A relevância dessa interface emergiu a partir dos processos de crescimento populacional nas últimas décadas e da consequente utilização e adaptação de recursos naturais, associados ao uso das tecnologias. Nesse contexto, a evolução do conhecimento sobre saúde e meio ambiente passou a ser uma peça-chave para gerar intervenções positivas de preservação dos recursos naturais do planeta e também da saúde dos organismos. Tanto as ações do Estado quanto as pesquisas e produção científica sobre o tema ambiente se intensificaram ao longo do século XX, em especial na área da saúde coletiva (FREITAS, 2005).

Na compreensão de Helman (2009), tratase de uma competência cultural que se tornou tão popular entre os planejadores de saúde, bem como entre médicos e enfermeiros, que foi utilizada para melhorar a sensibilidade dos profissionais da saúde às crenças culturais, práticas, expectativas e origens de seus pacientes e comunidades; melhorar o acesso aos cuidados de saúde, eliminando as barreiras estruturais aos cuidados de saúde de qualidade para as minorias; e reduzir as barreiras organizacionais, como o pequeno número de profissionais de saúde, administradores e colaboradores de políticas selecionados dentre das comunidades de minorias, os quais poderiam planejar serviços de saúde mais apropriados culturalmente.

No Brasil, o tema saúde e meio ambiente tem sido abordado em dissertações e teses de inúmeros programas de pós-graduação; entretanto, essa produção científica vem sendo pouco divulgada. Este estudo teve por objetivo caracterizar a produção científica brasileira sobre saúde e meio ambiente, mediante a avaliação das dissertações e teses de programas de pós-graduação strictu sensu que abordaram o tema no período de 1987 a 2010. Nessa avaliação, foram levados em conta fatores como número de trabalhos produzidos e sua distribuição em termos de áreas do conhecimento, regiões geográficas e instituições de ensino.

Num momento em que a discussão sobre a qualidade da produção científica no país, incluindo produções na área de saúde pública, surge como uma questão de debate, a sistematização dos dados, mencionados acima, pode indicar possibilidades de integração de diferentes pesquisas, ajudar a identificar duplicações ou contradições, a determinar lacunas não exploradas na interface saúde/ meio ambiente e a direcionar novas políticas públicas relacionadas a esse tema.

\section{Metodologia}

O estudo caracterizou-se como uma pesquisa bibliográfica, inventariante e descritiva, do tipo "estado da arte" ou "estado do conhecimento", desenvolvida com a finalidade de reunir e sintetizar dados de pesquisas de mestrado e doutorado sobre 
o tema saúde ambiental. Para a seleção da amostra, foram identificados os resumos de teses e dissertações, defendidas no período de 1987 a 2010, consultados a partir de uma única fonte (Banco de Teses da Coordenação de Aperfeiçoamento de Pessoal de Nível Superior - CAPES), no sentido de eliminar uma possível heterogeneidade entre materiais publicados em diferentes meios de divulgação (catálogos, teses, anais de eventos, entre outros). O ano de 1987 corresponde ao ano de lançamento do Banco de Teses da CAPES.

A opção pela análise de resumos foi baseada no fato desse tipo de produção apresentar os dados gerais de uma pesquisa e de possuir estrutura padronizada em termos de conteúdo (apresentam o objetivo, apontam o percurso metodológico e descrevem os resultados alcançados).

Esta pesquisa caracteriza-se como um estudo cienciométrico, realizado a partir de informações do Banco de Teses e Dissertações da CAPES. A busca junto ao site da CAPES estabeleceu as seguintes palavraschave: "meio ambiente e saúde", "saúde e meio ambiente", "ambiente e saúde" e "saúde ambiental". O levantamento dos dados foi realizado por meio da seleção das teses e dissertações que apresentavam no título, ou nas palavras-chave os termos citados anteriormente. Foram identificados inicialmente 1.090 resumos entre dissertações e teses. Destes, 479 apareciam repetidos, ou apenas, utilizavam as expressões saúde e meio ambiente, mas não tinham como escopo a investigação sobre o tema. Dessa forma, restaram 611 resumos para serem investigados.

As informações dos resumos (instituição, programa de pós-graduação, título do trabalho, autor, orientador, ano da defesa, tema, objetivos, metodologia e resultados) foram reunidas e organizadas em um banco de dados, construído no Microsoft Excel. Por meio de um processo de análise cienciométrica e de conteúdo (BARDIN, 1977), foi possível o mapeamento da produção em anos, por período, áreas do conhecimento (segundo a classificação adotada pela CAPES), regiões do país e a natureza jurídica das instituições, às quais os resumos estavam vinculados. Os temas pesquisados foram agrupados em categorias que emergiram a partir da análise de conteúdo. Para correlacionar alguns dos dados, foram montadas curvas de correlação e obtidos os valores de $\mathrm{R}^{2}$, utilizando-se o Microsoft Excel.

\section{Resultados}

Para o período em estudo, foram identificados e analisados 611 resumos, sendo 435 dissertações de mestrado acadêmico, 79 dissertações de mestrado profissional e 97 teses de doutorado (Figura 1).

Neste estudo, foram avaliados resumos produzidos desde 1987. Entretanto, foi constatado que os primeiros trabalhos acadêmicos, sobre saúde e meio ambiente no país datam de 1989 e foram realizados em nível de mestrado acadêmico. A partir da década de 2000, ocorreu um crescimento significativo nas produções sobre a temática, com um aumento expressivo, principalmente, no número de mestrados acadêmicos e surgimento dos primeiros trabalhos de mestrado profissional. Para os três níveis, a produção seguiu um modelo de crescimento exponencial, conforme Figura 1.

Foi observado que existe uma correlação linear entre o número de dissertações e teses específicas sobre a temática saúde e meio ambiente e o número total de dissertações e teses, defendidas no Brasil, no período avaliado $\left(\mathrm{R}^{2}=0,9392\right.$ e $\mathrm{R}^{2}=0,8745$, respectivamente).

Para identificar as áreas que pesquisaram sobre a temática saúde e meio ambiente, foi 
Figura 1 - Distribuição temporal das teses e dissertações sobre a temática saúde e meio ambiente, no período de 1989 a 2010 , realizadas junto aos Programas de Pós-Graduação stricto sensu brasileiros. Os números acima das barras indicam os valores absolutos de trabalhos em cada categoria

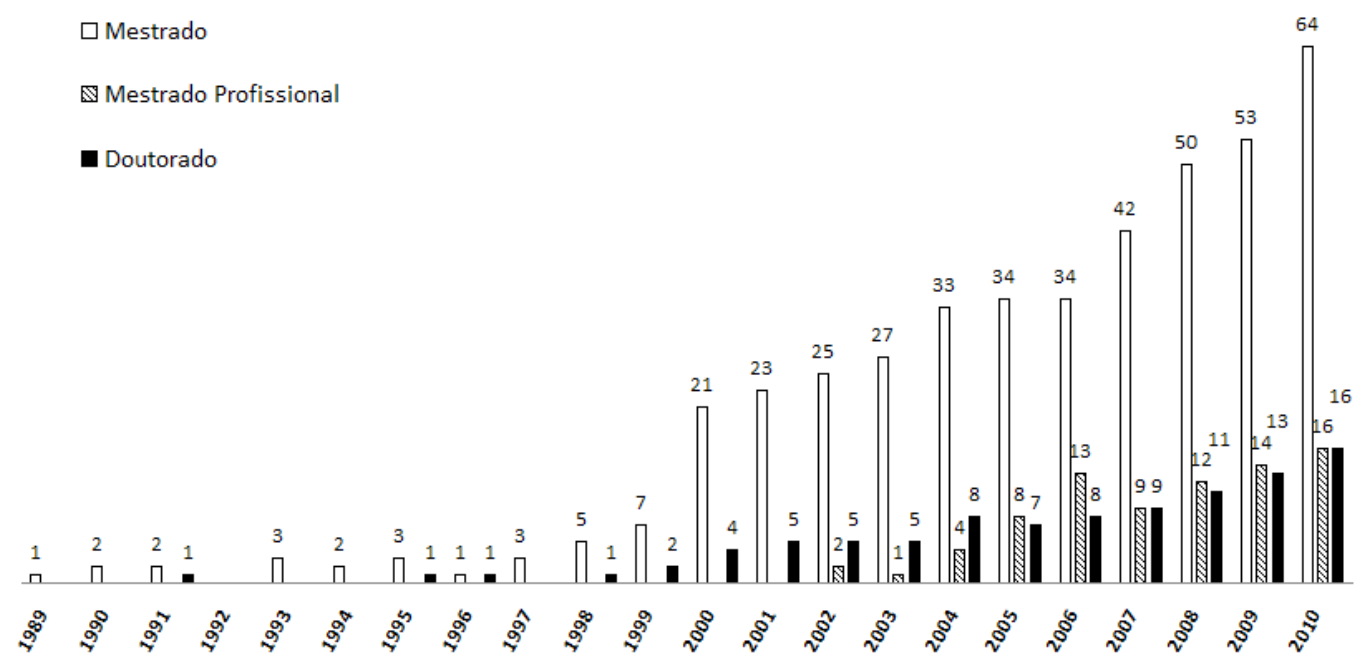

adotada a classificação proposta pela CAPES. Apareceram com destaque os trabalhos vinculados aos PPG das áreas de Ciências da Saúde e Multidisciplinar, seguido de estudos ligados às Engenharias (Tabela I).

A análise de conteúdo dos resumos das teses e dissertações possibilitou o agrupamento

Tabela I - Distribuição do número de dissertações e teses sobre a temática saúde e meio ambiente, desenvolvidas no período de 1989 a 2010, por grande área do conhecimento da CAPES

\begin{tabular}{|c|c|c|c|c|c|}
\hline \multirow{2}{*}{ GRANDES ÁREAS } & \multirow{2}{*}{$\begin{array}{c}\text { MESTRADO } \\
\text { strictu sensu } \\
\mathrm{N}^{\circ} \\
\end{array}$} & \multirow{2}{*}{$\begin{array}{c}\text { MESTRADO } \\
\text { PROFISSIONAL } \\
\mathbf{N}^{\circ} \\
\end{array}$} & \multirow{2}{*}{$\begin{array}{c}\text { DOUTORADO } \\
\mathbf{N}^{\circ} \\
\end{array}$} & \multicolumn{2}{|c|}{ TOTAL } \\
\hline & & & & $\mathrm{N}^{0}$ & $\%$ \\
\hline Ciências da Saúde & 152 & 12 & 46 & 210 & 34,3 \\
\hline Multidisciplinar & 137 & 37 & 22 & 196 & 32,1 \\
\hline Engenharias & 44 & 25 & 8 & 77 & 12,6 \\
\hline $\begin{array}{l}\text { Ciências Sociais } \\
\text { Aplicadas }\end{array}$ & 32 & 5 & 7 & 44 & 7,2 \\
\hline Ciências Humanas & 30 & 0 & 8 & 38 & 6,2 \\
\hline $\begin{array}{l}\text { Ciências Exatas e da } \\
\text { Terra }\end{array}$ & 20 & 0 & 0 & 20 & 3,3 \\
\hline Ciências Biológicas & 12 & 0 & 5 & 17 & 2,8 \\
\hline Ciências Agrárias & 8 & 0 & 1 & 9 & 1,5 \\
\hline $\begin{array}{l}\text { Linguística, Letras e } \\
\text { Artes }\end{array}$ & 0 & 0 & 0 & 0 & 0,0 \\
\hline Total Geral & 435 & 79 & 97 & 611 & 100 \\
\hline
\end{tabular}

*Áreas segundo a classificação proposta pela CAPES 
dos assuntos abordados em sete grandes temas (Tabela II). As questões relacionadas ao saneamento ambiental foram priorizadas nas pesquisas de mestrado e doutorado, estando presentes em $29,62 \%$ do total de teses e dissertações elaboradas. Em seguida, destacaram-se as áreas de educação $(19,15 \%)$ e de políticas públicas (16,20\%) (Tabela II).

As pesquisas sobre saúde e meio ambiente no país se concentraram principalmente em PPG de instituições localizadas na região sudeste $(57,77 \%)$, seguidos de PPG das regiões sul e norte $(15,38 \%$ e $15,05 \%$, respectivamente) (Figura 2A). Do total de trabalhos avaliados, aproximadamente $62,35 \%$ citaram, claramente no resumo, o local onde foi realizada, efetivamente, a pesquisa de campo, e foi observado que este local nem sempre coincidia com a região-sede do PPG. Por exemplo, a região nordeste, que apresentou o menor número de PPG, com trabalhos ligados ao tema em questão, é o segundo local de realização com maior número de estudos de campo (Figura 2B).

Figura 2 - Distribuição, por regiões brasileiras, das teses e dissertações sobre a temática saúde e meio ambiente no Brasil, no período de 1989 a 2010. A) Distribuição das pro- duções científicas (teses e dissertações somadas) por regiões onde se localizavam os PPG stricto sensu. B) Distribuição por regiões onde foram desenvolvidos os estudos de campo sobre a temática. Na figura 2B, foram incluídos apenas os trabalhos que especificavam claramente, no resumo, o local da pesquisa de campo (totalizando 381 trabalhos). Os números acima das barras indicam os valores absolutos em cada categoria
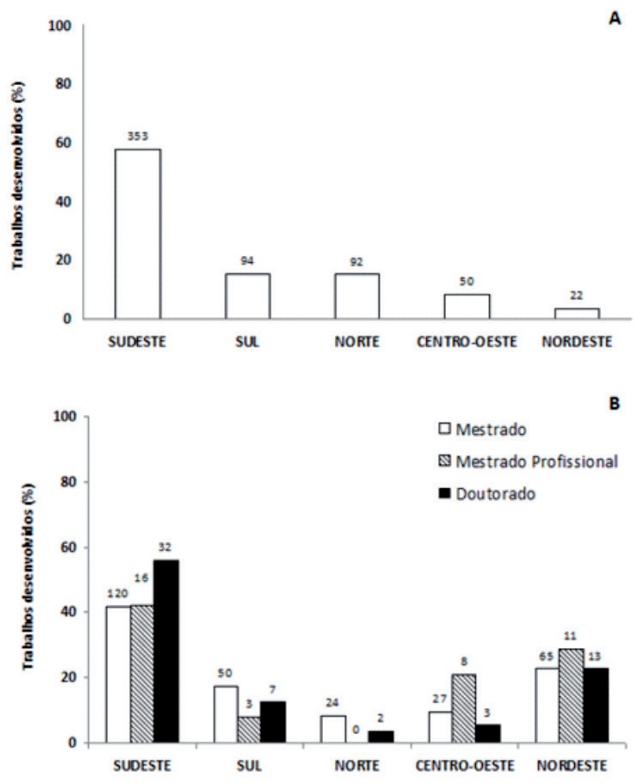

Em termos da natureza jurídica das instituições que realizaram pesquisas sobre

Tabela II - Temas contemplados nas pesquisas sobre saúde e meio ambiente, no período de 1989 a 2010, realizadas junto aos Programas de Pós-Graduação Stricto Sensu

\begin{tabular}{|c|c|c|c|c|c|}
\hline \multirow[t]{2}{*}{ TEMAS } & $\begin{array}{l}\text { MESTRADO } \\
\text { strictu sensu }\end{array}$ & $\begin{array}{l}\text { MESTRADO } \\
\text { PROFISSIONAL }\end{array}$ & DOUTORADO & \multicolumn{2}{|c|}{ TOTAL } \\
\hline & $\mathrm{N}^{0}$ & $\mathrm{~N}^{\mathbf{0}}$ & $\mathbf{N}^{\mathbf{o}}$ & $\mathbf{N}^{0}$ & $\%$ \\
\hline Saneamento ambiental & 134 & 25 & 22 & 181 & 29,62 \\
\hline $\begin{array}{l}\text { Alimentação saúde e meio } \\
\text { ambiente }\end{array}$ & 06 & 00 & 04 & 10 & 1,64 \\
\hline Poluição ambiental e saúde & 40 & 02 & 10 & 52 & 8,51 \\
\hline $\begin{array}{l}\text { Questões agrárias, saúde e } \\
\text { meio ambiente }\end{array}$ & 27 & 05 & 10 & 42 & 6,97 \\
\hline Educação & 85 & 16 & 16 & 117 & 19,15 \\
\hline $\begin{array}{l}\text { Políticas públicas, saúde e } \\
\text { meio ambiente }\end{array}$ & 71 & 11 & 17 & 99 & 16,2 \\
\hline Outros temas & 72 & 20 & 18 & 110 & 18,00 \\
\hline Total Geral & 435 & 79 & 97 & 611 & 100 \\
\hline
\end{tabular}


saúde e meio ambiente, o destaque é para as instituições públicas federais que produziram, praticamente, metade das dissertações e teses (Figura 3).

Figura 3 - Distribuição das dissertações e teses sobre saúde e meio ambiente, segundo a natureza jurídica das Instituições de Ensino Superior onde foram realizadas, no período de 1989 a 2010. Os números acima das barras indicam os valores absolutos de trabalhos em cada categoria

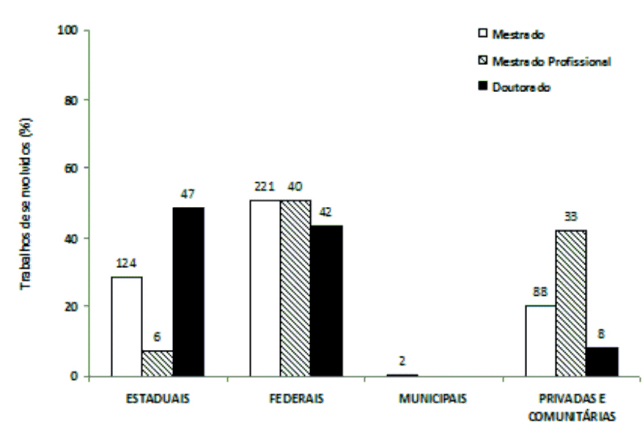

Em seguida, destacam-se as instituições públicas estaduais, com uma produção significativa, principalmente em nível de doutorado $(48,45 \%)$ e mestrado acadêmico $(28,51 \%)$. A produção total das instituições públicas federais e estaduais, incluindo dissertações e teses, foi de $78,56 \%$ da totalidade, contra $21,11 \%$ das privadas e comunitárias, e apenas $0,33 \%$ das municipais. Novamente, destacam-se as instituições federais da região sudeste e, mais especificamente, do estado de São Paulo. Nas universidades de São Paulo, foram desenvolvidas $15,05 \%$ das teses e dissertações sobre saúde e meio ambiente, seguidas pelas IES do Rio de Janeiro, com $8,02 \%$.

\section{Discussão}

\section{Número e distribuição dos trabalhos produzidos}

Os dados obtidos, no presente estudo, demonstram que houve um aumento significativo $(73,96 \%)$ no número de pesquisas sobre o tema saúde e meio ambiente a partir dos anos 2000 (Figura 1). Esse aumento coincidiu com um período de expansão do Sistema Nacional de Pós-Graduação (SNPG). Para exemplificar, entre 1998 e 2010, o número de PPG no país aumentou $125,6 \%$, e o número de bolsas, stricto sensu, concedidas pela CAPES aumentou 175,6\% (CAPES, 2014), o que demonstra que houve um forte investimento nacional para a capacitação de pessoal em nível de pós-graduação.

Nesse contexto, é razoável inferir que o crescimento das pesquisas sobre saúde e meio ambiente no Brasil é, pelo menos em parte, reflexo da expansão do SNPG. De fato, o número de dissertações e teses específicas sobre a temática saúde e meio ambiente e o número total de dissertações e teses defendidas no Brasil no período avaliado, correlacionam-se de forma linear $\left(\mathrm{R}^{2}=0,9392\right.$ e $\mathrm{R}^{2}=0,8745$, respectivamente, para dissertações e teses). Por outro lado, a evolução no número de trabalhos específicos sobre essa temática, em PPG de diferentes áreas do conhecimento, provavelmente foi, também, consequência de discussões ocorridas, a partir da década de 1990, sobre a relevância das relações entre saúde e ambiente.

Tais discussões aprofundaram a compreensão de que vivemos em uma sociedade de riscos disseminados globalmente. A globalização econômica, que está aliada à globalização política, cultural, informacional e comunicativa, traz novas oportunidades e desafios, cujos benefícios e impactos adversos, que envolvem todas as dimensões das relações humanas. Processos de interdependência planetária trazem benefícios e riscos para a saúde humana, de forma diferenciada ao redor do globo (FORTES; RIBEIRO, 2014).

Para Boaventura de Souza Santos (2020), uma das prováveis causas das pandemias são as relações autocentradas dos mercados sobre o social e sobre o ecológico. Esse modelo de 
exploração sem limites dos recursos naturais, vem conduzindo a humanidade a uma situação de catástrofe ecológica.

Essa exploração está a violar de maneira fatal o lugar da humanidade no planeta Terra. Esta violação traduz-se na morte desnecessária de muitos seres vivos da Mãe Terra, nossa casa comum, como defendem os povos indígenas e camponeses de todo o mundo, hoje secundados pelos movimentos ecologistas e pela teologia ecológica. Essa violação não ficará impune. As pandemias, tal como as manifestações da crise ecológica, são a punição que sofremos por tal violação. Não se trata de vingança da Natureza. Trata-se de pura auto-defesa. O planeta tem de se defender para garantir a sua vida. A vida humana é uma ínfima parte $(0,01 \%)$ da vida planetária a defender (SANTOS, 2020, p. 23).

Mas em termos de cuidado, a globalização, pode apresentar a propagação de informações para propor saídas e projetos úteis para a sociedade. Dessa forma, temas como urbanização descontrolada, insuficiência dos serviços básicos de saneamento, coleta e destinação adequada do lixo e condições precárias de moradia, tradicionalmente relacionados com a pobreza e o subdesenvolvimento, a partir dos anos 1990 passaram a ser discutidos juntamente com poluição do ar, da água, dos solos (ROHLFS et al., 2011).

As discussões para a incorporação das questões do meio ambiente nas políticas de saúde e a integração dos objetivos da saúde ambiental como estratégia de desenvolvimento sustentável, ganharam força tanto no cenário nacional quanto no internacional. Documentos internacionais, dentre eles a Agenda 21 Global, passaram a ressaltar a necessidade de uma abordagem mais integrada e intersetorial na busca de melhores condições de vida (UNESCO, 2005). Essas discussões deram maior visibilidade ao tema saúde e meio ambiente e, muito provavelmente, influenciaram a concepção e desenvolvimento de trabalhos de pós-graduação no Brasil.

\section{Áreas do conhecimento que pesquisaram sobre a temática}

O maior número de trabalhos sobre saúde e meio ambiente no Brasil foi desenvolvido por PPG das grandes áreas de ciências da saúde e multidisciplinar (Tabela I). Dados da CAPES demonstram que a área multidisciplinar foi uma das que mais cresceram no contexto da pós-graduação brasileira (aumento de $151,3 \%$ de 2004 para 2009), enquanto que as ciências da saúde concentraram o maior número de cursos (BRASIL, 2010). Dessa forma, novamente é possível concluir que a expansão das pesquisas sobre saúde e meio ambiente reflete, ao menos, parcialmente, à dinâmica do SNPG.

A grande vinculação de pesquisas sobre saúde e meio ambiente com PPG da área de ciências da saúde, contribuiu para fortalecer o conceito de saúde ambiental que vinha sendo incorporado às políticas públicas brasileiras da época. Nessa perspectiva, a saúde humana é entendida de uma forma mais global, vinculada às condições do meio (natural, cultural, social, econômico) em que as pessoas vivem, de forma que a promoção à saúde está ligada, fundamentalmente, à promoção de "ambientes saudáveis” (BRASIL, 2009). Capra e Luisi (2014) falam da necessidade de uma educação para a saúde, na qual o objetivo será o de fazer as pessoas compreenderem como o seu comportamento e o ambiente onde vivem, afetam sua saúde, e ensiná-las a lidarem com o estresse da vida diária.

Na grande área multidisciplinar, os trabalhos foram realizados especialmente por PPG pertencentes à área interdisciplinar, ou seja, por PPG que atuam em temas que exigem: 
[...] convergência de duas ou mais áreas do conhecimento, não pertencentes à mesma classe, que contribua para o avanço das fronteiras da ciência e tecnologia, transfira métodos de uma área para outra, gerando novos conhecimentos e disciplinas e faça surgir um novo profissional com um perfil distinto dos existentes, com formação básica sólida e integradora (BRASIL, 2010, p. 135)

A definição acima se ajusta à visão integradora inerente ao conceito de saúde ambiental. Isso demonstra que boa parte das pesquisas sobre saúde e meio ambiente desenvolvidas no país, se enquadram adequadamente à área de Pós-Graduação, à qual estão vinculadas.

A grande área de engenharias também produziu um número significativo de trabalhos de mestrado e doutorado sobre saúde e meio ambiente (Tabela I). As pesquisas, nessa grande área, estão concentradas principalmente na engenharia civil, de transportes, produção, sanitária e mecânica (dados não mostrados). Essa distribuição diversificada dos trabalhos científicos, entre diferentes engenharias, pode ser considerada como um reflexo da relação estreita entre saúde, meio ambiente e os setores produtivos da sociedade, como o setor industrial, o de construção civil, entre outros (SCHNEIDER, 2000; CARVALHO et al., 2001; BORCHARDT, 2008).

A inter-relação entre saúde, meio ambiente e engenharia pode promover o desenvolvimento prático de estratégias de melhoria da qualidade de vida. No caso específico do saneamento, a engenharia pode realizar intervenções no ambiente físico, voltadas a evitar a transmissão de doenças e assegurar a salubridade do ambiente. Os engenheiros também são responsáveis por implantar adaptações tecnológicas que atendem às características físicas da área, bem como responsáveis por ações voltadas a educação, novos hábitos e costumes da população (SOUZA; FREIRAS, 2010).

Um aspecto interessante observado foi que, excetuando-se Linguística, Letras e Artes, todas as demais grandes áreas do conhecimento desenvolveram trabalhos vinculados à saúde e ao meio ambiente, em maior ou menor proporção (Tabela I). Como já mencionadas, as questões ambientais ganharam destaque, nas últimas décadas, em diferentes esferas dos setores público e privado, tanto em nível nacional quanto internacional (BRASIL, 2009; ROHLFS et al., 2011; UNESCO, 2005). Os resultados; aqui apresentados, podem ser consequência da influência de tais discussões sobre o meio acadêmico brasileiro como um todo. Essa mudança de mentalidade para uma visão mais integrativa e associativa das diferentes áreas, está afinada com o que está ocorrendo na área da saúde que, também; está migrando de uma abordagem biomédica de assistência à saúde para uma abordagem sistêmica e integrativa, fortalecendo ainda muito mais as possibilidades de planejamento de estudos, estratégias e ações conjuntas entre as diferentes áreas com a afinidade de propósito.

\section{Temas priorizados nas pesquisas}

A maior parte das pesquisas de mestrado e doutorado sobre saúde e meio ambiente priorizou como tema central questões relacionadas com o saneamento (Tabela II). Essa escolha se explica em termos históricos, pois a influência do saneamento sobre a saúde desencadeou as primeiras discussões sobre saúde e meio ambiente (HELLER, 1998). A compreensão das relações entre essas áreas (saneamento, saúde pública e meio ambiente), fez com que, nas últimas décadas, os projetos de saneamento tenham saído de sua 
concepção sanitária clássica, para recaírem em uma abordagem ambiental, que visa a não só promover a saúde do homem, mas também conservar o meio físico e biótico (SOUZA; FREIRAS, 2010). Essa nova compreensão determinou a implantação de políticas públicas (BRASIL, 2009) e provavelmente também, influenciou a realização de pesquisas de Pós-Graduação sobre a temática no Brasil.

Seguindo essa mesma abordagem, o número expressivo de produções vinculadas especificamente ao tema políticas públicas, saúde e meio ambiente (Tabela II), pode estar associada a uma visão mais integrada da infraestrutura das cidades, na qual há uma complementaridade entre gestão pública, habitação, saneamento, transporte e atenção básica de saúde. Segundo Helman (2009), o sistema dominante de cuidados de saúde de qualquer sociedade não pode ser estudado isoladamente de outros aspectos dessa sociedade, pois o sistema médico (ou profissional dos cuidados de saúde) não existe em um vácuo cultural. Ao contrário, ele é uma expressão e, em certa medida, um modelo em miniatura dos valores e da estrutura social da qual se origina.

A educação também foi um tema bastante recorrente nos resumos das dissertações e teses avaliados. Esse aspecto é interessante, tendo em vista a importância da educação para a efetivação de práticas sociais e culturais de promoção ao meio ambiente e, por consequência, de promoção à saúde. A visão integrada de educação e promoção à saúde se contrapõe à visão de criar políticas públicas destinadas, exclusivamente, aos doentes ou instituições de tratamento. Modelos de gestão em saúde mais inovadores, que tratam da defesa efetiva dos direitos humanos, são favoráveis ao acesso da população a serviços de saúde e educação, bem como ao manejo sustentável de recursos naturais (BRASIL, 2009; BAENINGER, 2010).

\section{Distribuição geográfica dos PPG que produziram as pesquisas}

Mais de $50 \%$ das pesquisas sobre saúde e meio ambiente, no país, foram desenvolvidas na região sudeste (Figura 2A). Perfil similar foi observado em estudos que avaliaram a produção científica sobre outras temáticas, como o saneamento (SOUZA; FREIRAS, 2010), ambiente na saúde coletiva (FREITAS, 2005) e gênero e saúde (AQUINO, 2006). Tais dados refletem, novamente, a tendência de centralização dos PPG no país, que pode resultar na consequente centralização da geração de conhecimento não só sobre saúde e meio ambiente, mas também sobre outros temas, igualmente, relevantes.

O perfil centralizado da produção científica pode ter influência sobre o desenvolvimento socioeconômico nas diferentes regiões do país. Pesquisas indicam que há uma relação entre a massa crítica, atuante em Ciência e Tecnologia (C\&T) no Brasil, e o PIB por região (CASALI et al., 2010). A renda per capita das regiões norte e nordeste é menos da metade do observado na região sudeste (PINO, 2012) e, historicamente, a região sudeste concentra o maior número de grupos de pesquisa, no país, e também o maior Produto Interno Bruno - PIB (CNPq, 2014; IPECE, 2012).

Em função dessas discrepâncias, o país adotou uma política, para atenuar o desequilíbrio regional, na formação de recursos humanos, com investimentos diretos nas regiões norte, nordeste e centro-oeste, e destinação de $30 \%$ dos editais para essas regiões. Tal política resultou em um processo inicial de desconcentração da formação de mestres e doutores no Brasil. Assim, a região sudeste diminui sua contribuição relativa no número de PPG stricto sensu brasileiros, de 61,39\%; em 1998, para 48,62\%, em 2010 (CNPq, 2014; CAPES, 2014). 
Esse processo de descentralização parece, ainda, não ter tido um reflexo tão direto nas pesquisas específicas sobre saúde e meio ambiente. Para exemplificar, é possível comparar as regiões sudeste e nordeste que diferem, significativamente, em número absoluto de trabalhos gerados sobre a temática. Em ambas, o pico de dissertações defendidas sobre saúde e meio ambiente foi atingido em 2009; porém, na região sudeste, o número absoluto de trabalhos, no referido ano, foi de 45 , enquanto que, no nordeste foi de apenas quatro.

\section{Locais de campo onde as pesquisas foram desenvolvidas}

A região sudeste também foi a região na qual foi desenvolvido, efetivamente, o maior número de trabalhos sobre saúde e meio ambiente (Figura 2B), indicando que a tendência de concentração dos locais de realização das pesquisas está, também, relacionada à tendência de concentração das instituições que oferecem mestrado e doutorado, nas quais estudantes de todas as regiões do país buscam espaço de formação.

Entretanto, é importante considerar que alguns estudantes desenvolveram suas pesquisas (principalmente estudos de campo), provavelmente em seus locais de origem (estados, cidades, bairros, instituições) e não nas regiões onde os PPG estão situados. Esse fato é notável, especialmente, no caso da região nordeste, a qual apresentou o menor número de dissertações e teses sobre saúde e meio ambiente, defendidas no período de análise, mas foi o segundo maior "local-alvo" das pesquisas realizadas (Figura 2A e 2B).

\section{Natureza das instituições nas quais foram realizadas as pesquisas}

A maioria das pesquisas sobre saúde e meio ambiente, no Brasil, foram desenvol- vidas em instituições de ensino federais ou estaduais, com um número reduzido de trabalhos realizados em instituições municipais, privadas ou comunitárias, principalmente em nível de doutorado (Figura 3). Isso provavelmente ocorreu em razão de haver maior número de PPG em instituições federais ou estaduais.

Em números absolutos, as instituições públicas federais e estaduais são as que mais contribuíam para a formação de mestres e doutores no país. Entretanto, na última década da pesquisa, as instituições privadas aumentaram sua participação no sistema nacional de pós-graduação, elevando sua parcela na formação de mestres: de 13,3\%, para $22,4 \%$ (entre 1996 e 2009) e doutores, de $7,3 \%$ para $9,5 \%$ (entre 1996 e 2008) (CGEE, $2010 ; 2012$ ). Tal fato pode ter reflexo futuro nos trabalhos sobre saúde e meio ambiente, os quais, em grande parte, são vinculados à área multidisciplinar (Tabela I), que é uma das áreas onde se concentra boa parte dos PPG das instituições privadas (CGEE, 2012). Considerando-se que a implantação de cursos de doutorado é uma exigência para a manutenção do status de universidade, é provável que as instituições privadas aumentem ainda mais sua participação no SNPG e, por consequência, na produção sobre saúde e meio ambiente.

No caso do mestrado profissional, a contribuição das universidades privadas, na produção científica sobre saúde e meio ambiente, praticamente se igualou à das instituições federais (Figura 3). O mestrado profissional é uma modalidade voltada para a formação de profissionais qualificados para o setor produtivo (CGEE, 2012). O aluno que ingressa nessa modalidade, muitas vezes já está inserido no mercado de trabalho e busca uma qualificação direcionada para seu ramo de atividade. Nessa situação, existe maior viabilidade financeira (o aluno não depende de bolsas de estudos) e de tempo (o aluno 
em geral pode desenvolver a pesquisa no seu local de trabalho), facilitando a vinculação em PPG particulares. De fato, dos resumos de mestrado profissional analisados neste trabalho, 50\% mencionavam o espaço de realização das pesquisas ( 27 resumos) e, destas, $51,85 \%$ foram realizadas em empresas.

\section{Considerações Finais}

Nesse estudo, foi realizada uma análise das produções científicas brasileiras (dissertações e teses) que abordaram o tema saúde e meio ambiente, no período de 1989 a 2010, apresentando um panorama geral sobre esse tema. Os resultados mostram que tais pesquisas seguem algumas tendências relacionadas com as características do próprio SNPG como: I) grande expansão nas últimas décadas e II) centralização em regiões/estados do sudeste do país. Adicionalmente, a produção científica nacional sobre saúde e meio ambiente está alinhada com o conceito contemporâneo de saúde ambiental, o qual embasa a visão atual de políticas públicas em meio ambiente e saúde no Brasil.

Os principais $\mathrm{PPG}$, que realizaram pesquisas sobre a temática saúde e meio ambiente, pertencem às áreas de ciências da saúde e multidisciplinar. Os temas mais recorrentes nessas produções são relacionados ao saneamento, políticas públicas e educação. Grande parte dos trabalhos foi desenvolvida em PPG localizados no sudeste do Brasil, fato, possivelmente, relacionado à tendência supracitada de concentração das instituições que oferecem mestrado e doutorado nessa região. As universidades federais e estaduais são as que mais desenvolveram pesquisas sobre a temática, sendo que, apenas, uma pequena parcela dos estudos foi realizada em instituições municipais, particulares ou comunitárias. Nesse último caso, destaca-se a contribuição nos mestrados profissionais.
Diante dos resultados aqui expostos, verifica-se que, no Brasil, no período do estudo, os trabalhos relacionados ao tema saúde e meio ambiente envolvem subtemas e áreas do conhecimento diversas que, direta ou indiretamente, buscam: I) diminuir os riscos à saúde, decorrentes da exposição a contaminantes ambientais, II) melhorar a vigilância em saúde, e III) melhorar a qualidade de vida da população. O contexto apresentado confirma a complexidade inerente ao tema e reforça a necessidade de pesquisas multidisciplinares e ações intersetoriais no campo da gestão pública. Assim, justificam-se incentivos à ampliação do número de PPG, ou grupos de pesquisa, que tratem da temática, bem como uma descentralização das pesquisas, no sentido de atender às demandas nacionais e regionais de integração entre sustentabilidade, ambiente e saúde.

A evolução a respeito do conceito do que é saúde criou novas formas de atender e intervir, nessa área, com políticas e abertura de novos campos de estudos para solução de problemas que se apresentavam aos pacientes, a partir da compreensão de saúde não mais como completo estado de bem-estar, mas como equilíbrio dinâmico; este implica que a doença é uma consequência de desiquilíbrio e de desarmonia, que pode surgir em vários níveis do organismo e pode gerar sintomas de natureza física, psicológica ou social. Em relação ao meio ambiente, embora, teoricamente, se tenha conhecimento e se saiba de sua relevância, das consequências e impactos de sua degradação, parece que o ser humano, do ponto de vista prático, o vê como algo distante, como se não lhe dissesse respeito, sempre fosse algo que se referisse aos outros, "não lhe pertencesse". Em aspectos lesivos, relacionados à saúde, as pessoas sentem como uma perda "sua" no tocante ao meio ambiente, as degradações não consideram algo "seu". Basta ver a importância que dão a campanhas de prevenção, anúncios ou 
até doações. E isso está, diretamente, relacionado à forma como o ser humano vai cuidar do meio ambiente. Ele não compreendeu que o mundo é a sua casa e condição de sua sobrevivência, bem como que sua saúde tem relação direta com o ambiente em que vive.

Essa consciência e visão integrada the faltam e, talvez, seja um aspecto pouco reforçado pelas campanhas publicitárias da área da saúde, e também pelos profissionais que atuam como médicos, fisioterapeutas, farmacêuticos, enfermeiros, que direcionam mais o olhar para a saúde curativa, para o paciente, secundarizam o ambiente onde ele vive ou, quem sabe, a grande maioria ainda foi formado dentro do modelo biomédico. Isso se torna mais claro quando pensamos que o atendimento de quem adoece é feito em hospitais e postos de saúde, longe das casas ou bairros, locais onde as pessoas moram, muitas vezes. Os profissionais da saúde não têm sequer a mínima noção de como vivem os pacientes, ou para onde vão voltar depois de saírem, de terem sido internados de um hospital com pneumonia, por exemplo. A relação saúde-meio ambiente nem é lembrada. Portanto, isso demonstra o quanto há para se pensar e fazer em um país como o Brasil, que apresenta índices alarmantes de esgoto a céu aberto nas cidades, apenas para citar um dos tantos problemas para serem solucionados, sem contar o retorno de doenças que já haviam sido erradicadas, como a catapora. Mas não há outro caminho que não seja o do conhecimento, da conscientização, da criação de políticas públicas eficazes e sua implementação para solucionar os problemas. É assim que se encontram as saídas.

\section{REFERÊNCIAS}

AQUINO, E. Gênero e saúde: perfil e tendências da produção científica no Brasil. Rev Saúde Pública, v. 40, p. 121-132, 2006.

BAENINGER, R. População e Cidades: subsídios para o planejamento e para as políticas sociais. Campinas: Núcleo de Estudos de População, Nepo/Unicamp, Brasília: UNFPA, 2010.

BARDIN, L. Análise de conteúdo. Lisboa: Edições 70, 1977.

BASTOS, F. Saúde em questão. Rio de Janeiro: Fiocruz, 2011.

BORCHARDT, M; POLTOSI, L. A. C.; SELLITTO PEREIRA, A. M.; MEDEIROS, G.

Considerações sobre ecodesign: um estudo de caso na indústria eletrônica automotiva. Ambiente \& Sociedade, v. XI, n.2, p.341-353, 2008.

BRASIL. Ministério da Saúde. Conselho Nacional de Saúde. Subsídios para construção da Política Nacional de Saúde Ambiental. Brasília: Ministério da Saúde, 2009.

BRASIL. Ministério da Educação. Coordenação de Aperfeiçoamento de Pessoal de Nível Superior. Plano Nacional de Pós-Graduação - PNPG 2011-2020. Brasília: CAPES, 2010.

BUSS, P. M. Promoção da saúde e qualidade de vida. Ciênc. Saúde Coletiva, v. 5, n. 1, p. 163-177, 2000.

CAMARGO, K. R. Produção científica: avaliação da qualidade ou ficção contábil. Cad Saúde

Pública, v. 29, n. 9, p. 1707-1711, 2013. 
CAMARGO, L. M. A. Epidemiological aspects of American cutaneous leishmaniasis and phlebotomine sandfly population, in the municipality of Monte Negro, State of Rondônia. Revista da Sociedade Brasileira de Medicina Tropical, v. 46, n. 1, p. 60-66, 2013.

CAPES. Dados GeoCAPES. Disponível em: http://geoCAPES.CAPES.gov.br/. Acesso em: 14 fev. 2014.

CARVALHO, B G.; MARTIN, G. B.; CORDONI JÚNIOR, L. A organização do sistema de saúde no Brasil. In: ANDRADE, S. M.; SOARES, D. A.; CORDONI JÚNIOR, L. (Org.). Bases da saúde coletiva. Londrina: Ed. UEL, 2001. p.27-59

CORDONI JÚNIOR, L. (Org.). Bases da saúde coletiva. Londrina: Ed. UEL, 2001. p.27-59.

CARVAlHO, M. S.; TRAVASSOS, C.; COELE, C. Medicina. More of the same epidemiology.

Cadernos de Saúde Pública, v.29, n.11, p. 21-41, 2013.

CARTER-POKRAS, O.; ZAMBRANA, R.; POPPELL, C.; GUERRERO-PRESTON, R. The environmental health of Latino children. J Pediatr Health Care, v.21, p.307-314, 2007.

CASALI, G. R.; SILVA, O. M.; CARVALHO, F. Sistema regional de inovação: estudo das regiões brasileiras. Revista de Economia Contemporânea, v.14, n.3, p.515-550, 2010.

CGEE. Centro de Gestão e Estudos Estratégicos. Doutores 2010: Estudos da demografia da base técnico-científica brasileira. Brasília: CGEE; 2010.

CGEE. Centro de Gestão e Estudos Estratégicos. Mestres 2012: Estudos da demografia da base técnico-científica brasileira. Brasília: CGEE; 2012.

CNPq. Dados Censos. 2014 Disponível em: http://dgp.cnpq.br/censos/. Acesso em 14 de fev. 2014. CONFALONIERI, U. Saúde na Amazônia: um modelo conceitual para a análise de paisagens e doenças. Estudos avançados, v.19, n.53, p.221-236, 2005.

FORTES, P. A. de C.; RIBEIRO, H. Saúde Global em tempos de globalização. Saúde e Sociedade, v.23, n.2, p. 366-375, 2014.

FREITAS, C. M. A produção científica sobre o ambiente na saúde coletiva. Cad Saúde Pública, v.21, n.3, p.679-701, 2005.

GOMES, L. P.; ESTEVES, R. V. R. Análise do sistema de gerenciamento dos resíduos de serviços de saúde nos municípios da bacia hidrográfica do Rio dos Sinos, Rio Grande do Sul, Brasil.

Engenharia Sanitária e Ambiental, v.17, n.4, p. 377-384, 2012.

HELLER, L. Relação entre saúde e saneamento na perspectiva do desenvolvimento. Ciênc. Saúde Coletiva, v.3, n.2, p.73-84, 1998.

IPECE. Instituto de Pesquisa e Estratégia Econômica do Ceará. A Evolução do PIB dos Estados e Regiões Brasileiras no Período 2002-2010: valores definitivos. Fortaleza: IPECE; 2012.

MINAYO, M. C. de S. Saúde e Ambiente: uma relação necessária. In: CAMPOS, Gastão Wagner de Sousa Campos et al. (org). Tratado de Saúde Coletiva. Rio de Janeiro: Hucitec, 2012, p.79-108.

MYERS, S.; LYNNE, G.; GOLDEN, C.; OSTFELD, R., REDFORD, Kent, RICKETTS, T., TURNER, Will; OSOFSKY, S. Human health impacts of ecosystem alteration. Proc Natl Acad Sci, USA, v. 110, n. 44, p. 18753-18760, 2013.

ORGANIZAÇÃO PAN-AMERICANA DA SAÚDE. Políticas integradas em rede e a construção de espaços saudáveis. Brasília: Vozes e Lugares, 2010.

PINO, B. A. Transformações globais, potências emergentes e Cooperação Sul-Sul: desafios para a cooperação europeia. Caderno CRH, v. 25, n. 65, p. 233-249, 2012. 
REDSHAW, C.; STAHL-TIMMINS, W.; FLEMING, L.; DAVIDSON, I.; DEPLEDGE, M. Potential changes in disease patterns and pharmaceutical use in response to climate change. Jornal Toxicol Environ Health B Crit Rev., v. 16, n. 5, p. 285-320, 2013.

ROHLFS, D. B.; GRIGOLETTO, J. C.; NETTO, G. F., RANGEL, C. F. A construção da vigilância em saúde ambiental no Brasil. Cad Saúde Coletiva, v.19, n. 4, p.391-398, 2011.

SCHNEIDER, S. Indústria, território e meio ambiente no Brasil: perspectivas da industrialização descentralizada a partir da análise da experiência catarinense. Revista Brasileira de Ciências Sociais, v.15, n.43, p.190-194, 2000.

SOUZA, C. M. N.; FREITAS, C. M. A produção científica sobre saneamento: uma análise na perspectiva da promoção da saúde e da prevenção de doenças. Engenharia Sanitária e Ambiental, v.15, n.1, p.65-74, 2010.

TELES, C. B. G.; BASANO, A. S.; ZAGONEL-OLIVEIRA, M.; CAMPOS, J. J.; OLIVEIRA, A. F. J.; FREITAS, R.A.; MEDEIROS, J. F.; PESSOA, F. A. C.; BARRAL, A.; CAMARGO, L. M. A. Epidemiological aspects of American cutaneous leishmaniasis and phlebotomine sandfly population, in the municipality of Monte Negro, State of Rondônia, Brazil. Revista da Sociedade Brasileira de Medicina Tropical, v. 46, n. 1, p.60-66, 2013.

UNESCO. Década da educação das nações unidas para um desenvolvimento sustentável, 20052014: documento final do esquema internacional de implementação. Brasília: UNESCO; 2005.

VEIGA, L. H. S.; FERNANDES, H. M. Avaliação de risco para a saúde humana e ecossistemas. In: BRILHANTE, O. M.; CALDAS, L. Q. de A. Gestão e avaliação de risco em saúde ambiental. Rio de Janeiro: FIOCRUZ, 1999. p.119-144. 
